\title{
Developing the concept of autonomous control of the quarry vehicles movement
}

\author{
Ivan Chicherin ${ }^{1 *}$, Boris Fedosenkov ${ }^{1}$, Dmitriy Dubinkin ${ }^{2}$, and Wang Zhenbo ${ }^{3}$ \\ ${ }^{1}$ T.F. Gorbachev Kuzbass State Technical University, Department of Information and Computer-aided \\ Manufacturing Systems, 650000 Kemerovo, 28 Vesennyaya st., Russian Federation \\ 2 T.F. Gorbachev Kuzbass State Technical University, Department of Metal-cutting Machines and \\ Tools, 650000 Kemerovo, 28 Vesennyaya st., Russian Federation \\ ${ }^{3}$ Shenyang University of Technology, Shenyang Mechanical and Electrical Engineering College, \\ 110870 Shenyang, Liaoning, No. 111 West Shenliao Road, Economic \& Technical Development \\ Zone, People's Republic of China
}

\begin{abstract}
Introduction. The article presents the results of studies related to the description of the synthesis and further analysis of the dynamic behavior of unmanned vehicles (UMVs) operating at coal opencast mines and other mining enterprises. The relevance of the research topic is dictated by the need to create safe and effective conditions for overburden / mining and mining transportation operations. This is ensured, among other things, by the operation of unmanned heavy-duty dump trucks. Purpose of the work: in the course of the research, a concept was developed for the formation of route parameters and models of the dynamics of movement of UMVs along technological routes. Methodology. The paper describes the technology for generating and processing signals in control subsystems as part of an automated dispatching system and an on-board subsystem for autonomous control of the UMV. Research methods include the study of the shapes and characteristics of current trajectories $(\mathrm{CT})$ in relation to a nominal axial trajectory (NAT). In this case, a current trajectory of the UMV is considered within the framework of the so-called S-frames, which are formed as geolocations on a certain working UMV route from a bench face to the place of unloading and vice versa. As a variable characterizing the UMV behavior on a current trajectory, a $1 \mathrm{D}$-signal with a timedependent frequency is adopted, the nature of the change of which is determined by the CT dynamic shape relative to the NAT. Results. Such chirp signals - together with the wavelet transforms technique introduced into consideration - allow us in a semantically transparent and information-rich videographic form to display and subject to further processing signals characterizing the current behavior of a UMV on a route. The resulting parameters of such secondary wavelet representations of the current UMV dynamics (wavelet maps) are further planned to be used in the subsystem of dynamic modal control of the UMV movement in the open pit. Conclusions. The results of the studies carried out make it possible to form current routes of UMV movement according to the models generated based on the
\end{abstract}

\footnotetext{
* Corresponding author: chiv.iap@kuzstu.ru
} 
analytical tools accompanying the dynamics of the UMVs, proposed in the work.

\section{Introduction}

The subject of research in the proposed work is the questions characterizing the dynamics of unmanned vehicles movement in the conditions of producing works of technology in coal open pits. The complexity of solving the problem is determined by the extremely difficult conditions for stripping / mining and transportation operations. The need to create safe and efficient working conditions in the transport segment of the «Smart quarry» system dictates the relevance of this kind of research [1-3]. Its relevance is confirmed by intensive research available: work on modeling and designing, creation of conditions and support systems for unmanned dump trucks operating in quarry conditions in many coal-mining countries all over the world.

\section{Methodology}

The Smart Quarry Management System (SQMS) is designed to implement the global functions of full-scale management of the structure of a coal open pit.

The central core of the "Smart quarry» management system is the computer-aided dispatching system (CDS).

The structure of the SQMS implies the presence of 2 functional purpose levels:

1) strategic level (covering all objects of the mining transportation complex);

2) tactical-operational level of local computer supporting the current state of unmanned vehicles (UMVs) as the subject of research.

To perform tasks at the strategic and tactical-operational levels, a module is used for dispatching a certain UMV current state, which is one of the SQMS fragments.

We will consider the solution of tasks for automating the functions of strategic and tactical-operational levels for management in the «Smart quarry» system below.

\section{Cartographic and photogrammetric works}

To achieve the goal of global management of the "Smart quarry", it is necessary to solve the following tasks to automate the functions of strategic and tactical-operational levels:

- setting the routes for the UMVs movement;

- monitoring the static and dynamic states of mining transportation equipment (MTE) which is in an active working phase;

- monitoring the state of the quarry administrative and economic complex structures;

- monitoring some objects and MTE that are in the state of reserve and repair in appropriate locations;

- management of the socio-economic sphere formed by the quarry administration.

To solve the problem of setting UMV routes in order to digitally enter them into the corresponding directories of computer-aided workstations (CWSs) of the control center (CC) as part of the SQMS, a method is proposed for forming digital ground locations of the routes planned that is based on mapping the quarry surface with using multi-rotor type un- 
manned aerial vehicles (UAVs) [4-7] equipped with the necessary equipment for such purposes. This task will be described in our further studies.

\section{Formation of spline frames for UMV movement routes}

Upon receipt of a quarry photo location, it is then transited to the external control subsystem within CC (CC ECSS). There, this material is subjected to interactive processing [8-11], the algorithm of which is as follows:

1) segmentation of the "Quarry" location into frames of an entire UMV route from the bench face to the unloading site / warehouse, that is, dividing each such global i-th location into local sub-routes of the corresponding technological route designation;

2) digital shaping each local sub-route, the shape of which is determined by the open pit bench width, the width of the route carriageway, and the UMV geometric dimensions. As a result, a few quasi-rectangular fragments with a given local width corresponding to a few local sub-routes are input into the ECSS;

3) digital fixing the sub-routes boundaries. Since the border lines (both the NAT line and the lateral border lines) are generally curvilinear segments, it is appropriate to call the generated fragments spline frames (S-frames);

4) introduction of photo maps of all S-frames in digital form into the database "Sframes DB" within SQMS ECSS.

Note that when performing a particular task of designing S-frames for the UMV quarry routes, only fixing, in one way or another, the coordinates of the ground control points (in our case, the coordinates of 4 corner points for each local S-frame from the set of S-frames in their database).

It is important to note that lidars are used to categorize and monitor some changes in the road surface and environment during the movement of UMV, as well as changes in the plan and profile of the open pit during its operation.

The ECSS and ACSS (autonomous control subsystem) function for automated search and capture / fixation of the UMV location under loading in the bench face relative to MTE (an excavator or a bucket loader) is implemented by means of the lidar-radar-sonar unit (LRSU) of the ACSS by the ECSS command "search / capture of the UMV-under-loading place". This procedure is carried out taking into account and on the basis of a set of regulated setting parameters that ensure an adequate mutual positioning of MT-mechanisms in the pair "UMV - excavator / bucket loader" in the k-th face.

After the end of the UMV loading process, the procedure for searching the position of the starting point of the nominal axial trajectory - NAT - within the local S-frame of the UMV exit out of the k-th bench face is performed according to reference points of the beginning boundary for the next adjacent frame. Note that local route S-frames are «stitched» using the DJI GO software package (made in China). In addition, the D-RTK-2 ground station, working together with the DJI «Phantom 4 Pro RTK» UAV, has the same capabilities.

\section{Description of the UMV movement route within S-frames}

The computer-aided dispatching system (CDS) records the moment the j-th UMV enters the "S-frame of exit out of the bench face" to exit from the k-th face and further move along this frame. Then the moment of the end of the j-th UMV movement within the "S-frame of the exit" and of the entry into the "S-frame of the i-th main route" for moving along the 1-th 
open pit bench is recorded. Further, CDS registers the moment when the UMV movement ends within the local "main S-frame" and UMV enters the local "S-frame of entry to the unloading place" (to the warehouse of finished product: coal / rock / auxiliary materials).

It is also necessary to record the moments of the beginning and end of the UMV unloading: a request from the control center to the operator at the unloading place about the UMV readiness for unloading - the operator's response; sending a signal from the control center to the UMV board about the beginning of unloading; a signal from the operator about the end of unloading).

After the end of the unloading operation, the CDS records the moments of UMV starting from the unloading point and entering the area of the local "S-frame of departure from the unloading place" of the i-th global route and supports UMV moving along this frame. Further, the CDS fixes the moment when the UMV movement ends (on the i-th global route) within the local "S-frame of departure from the unloading place" and enters the area of the local "S-frame of the main route" - movement within this frame. This operation is followed by the procedure for fixing the moment of the end of UMV moving within the local "S-frame of the main route" and entering the zone of the local "S-frame of entry into the bench face". After that, the moment of UMV stopping on the face bottom is recorded and the signal is sent from the ACSS to the control center; giving a permitting signal from the ECSS to the excavator board about the start of loading / reciprocal reception "Roger". Further, the ECSS and ACSS receive a feedback signal (FB) from the excavator / bucket loader driver about the completion of loading. The loading time is recorded in the ECSS, after which the permitting ECSS-signal is sent to the UMV board (to the ACSS) about the start of UMV movement and its entry into the "S-frame of exit out of the bench face" zone.

Then, in a closed cycle, the software implements repeating the stages of the algorithm for tracking the UMV on a given route by the external control subsystem (CC ECSS).

\section{Functional purpose of the autonomous control subsystem (ACSS)}

4.1 Ensuring the possibility of an adequate response to signals from on-board technical means of monitoring, control, signaling, protection, and blocking:

- lidar-radar-sonar unit;

- video cameras- for registration of the surrounding situation round about UMV on the main route and places of loading / unloading - as part of a video pentasystem; cameras on the UMV sides and one on top for communication with the global navigation satellite communication (GNSC) satellite / UAV and fixing obstacles located high above the road surface;

- on the state of the UMV monitor and control equipment and on-board electrical and mechanical units.

4.2 Provision of permanent communication with the external control subsystem (ECSS in the control center) - in order to perceive control signals from the ECSS and transmit feedback signals from the ACSS to the ECSS.

4.3 Ensuring the translation of control signals from the ECSS to the UMV actuating mechanisms (AMs) and power units, as well as the registration of signals from the primary converters (sensors) installed on the fragments of the control systems of the UMV working elements (chassis, wheel system, diesel unit, brake system, subsystems of cooling, oil lubrication, air conditioning, fuel resources registering, movement speed, automatic transmission position, etc.). 


\section{Functional purpose of the external control subsystem (ECSS)}

ECSS is a part of the SQMS and intended for monitoring and controlling the UMV-onroutes dynamics from the control center.

The functionality of the ECSS ensures the execution of the following operations.

5.1 Tracking and controlling all the UMV movement stages on technological routes $(i=$ $1, \mathrm{M}$, where $\mathrm{i}$ is the number of a technological route, that is, the movement of the UMV one or several - along the same pit bench; $j=1, N$, where $j$ is the UMV number for the $i$-th route). Therefore, the $\mathrm{j}$-th UMV number for the i-th route has the form "ji".

All UMVs available in the quarry park must be numbered and entered into the "UMV DB" database. Actual technological routes (TR) are also numbered and entered into the database "TR DB".

5.2 Monitoring and controlling the current state parameters for all fragments of the UMV on-board system ACSS and its power units:

5.2.1 ensuring the possibility of remote adjustment of the ACSS elements parameters by acting on it with the ECSS control signals from the control center;

5.2.2 implementation of the full range of ACSS functions because of the failure of its local control units for on-board monitoring / control facilities, that is, in such a case the execution of the procedure for transferring the ACSS functions to the external control subsystem ECSS.

5.3 Communication with the operators of the MTE (excavators, bucket loaders, drilling rigs, fuel stations, and other machines) at the bench face $\mathrm{k}$, where $\mathrm{k}=1, \mathrm{P}$ is the face number.

Thus, BPS (ijkl) is a UMV having $N o j$ on a route $N o i$, working at a bench face $N o k$ with an excavator No $l$.

\section{Modal control of the UMV movement along technological routes}

In the presented study, a dynamic modal control has been developed and used (a discussion of this type of control is planned to be presented in next publications), which, in contrast to the static one [12-14], operates with moving the controlled system poles constellation on the complex plane during a certain transient process, the latter is the UMV current trajectory $(\mathrm{CT})$ deviation from a nominal axial trajectory on a route.

Let us consider the principle of controlling the UMV movement along a quarry technological route.

Fig. 1 schematically depicts two deviating current trajectories - within a straight Sframe - indicating forward (deviating from the NAT) and reverse (returning to the NAT) $\mathrm{CT}$, that is, in terms of CDS signals - forward and reverse transient processes (TP). 


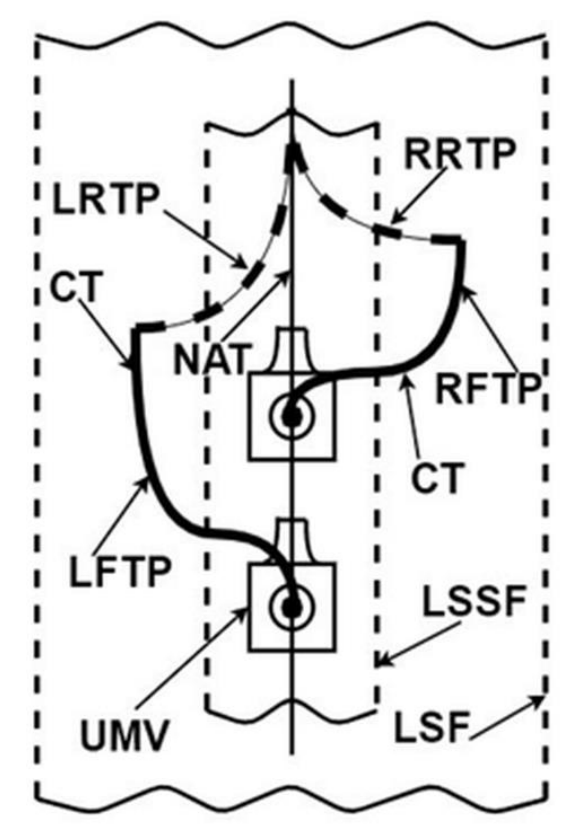

Fig. 1. The diagram of forming the UMV disturbed forward deviating paths and reverse restoring trajectories on the straight section of the S-frame of a particular route when the CT leaves to the left and right of the NAT

Fig. 1 shows the following notation:

- UMV - unmanned vehicles;

- LSF (Local Spline Frame) - local S-frame;

- LSSF (Local Sub-Spline Frame) - local sub-S-frame;

- NAT - nominal axial trajectory of the UMV movement;

- LFTP / RFTP (Left Forward Transient Process / Right Forward Transient Process) respectively, forward transient processes of the UMV movement current trajectory deviation to the left / right of the NAT;

- LRTP / RRTP (Left Reverse Transient Process / Right Reverse Transient Process) - respectively, reverse transient processes for the recovery of the NAT with the deviation of a UMV movement current trajectory (CT) to the left / right of the NAT.

By $\mathrm{x}(\mathrm{t})_{l}$ and $\mathrm{x}(\mathrm{t})_{r}$ we denote the generalized coordinate of the UMV movement when a trajectory deviates, respectively, to the left and right of the nominal axial trajectory in the direction of motion.

Fig. 2 in a combined form depicts curved S-frames of the UMV routes with current trajectories going to the left and right of the nominal axial trajectory. The points $\mathrm{A}_{l}$ and $\mathrm{B}_{l}$ denote the inflection points on the left forward and reverse trajectories, respectively; the point $\mathrm{C}_{l}$ is the inflection point on the right forward trajectory. $\mathrm{D}_{l}$ and $\mathrm{E}_{l}$ are the points indicating the activation of the modal control procedure on the left-handed and right-handed paths. In this case, the signal characterizing the UMV right reverse trajectory has the form of the impulse transient response of the 1st-order aperiodic curve [14]: 


$$
x(t)=w(t)=(k / T) \cdot \exp (-t / T)
$$

This waveform returns the CT to the NAT faster than the 2nd order aperiodic waveform. This prevents the UMV from hitting the side of the bench when moving along the route "bench face - warehouse - the bench side on the right", and falling from the bench on the route "bench face - warehouse - the bench side on the left".

When the TT is deflected to the left on the concave section of the S-frame, which also goes to the left, the return of the CT to the NAT under the action of modal control is made more smoothly - in order to avoid overshooting (crossing the NAT in the opposite direction) - according to the 2 nd order aperiodic curve. In this case, the lidar-radar-sonar unit continuously scans the surrounding environment, in particular, for the detection of an oncoming UMV.

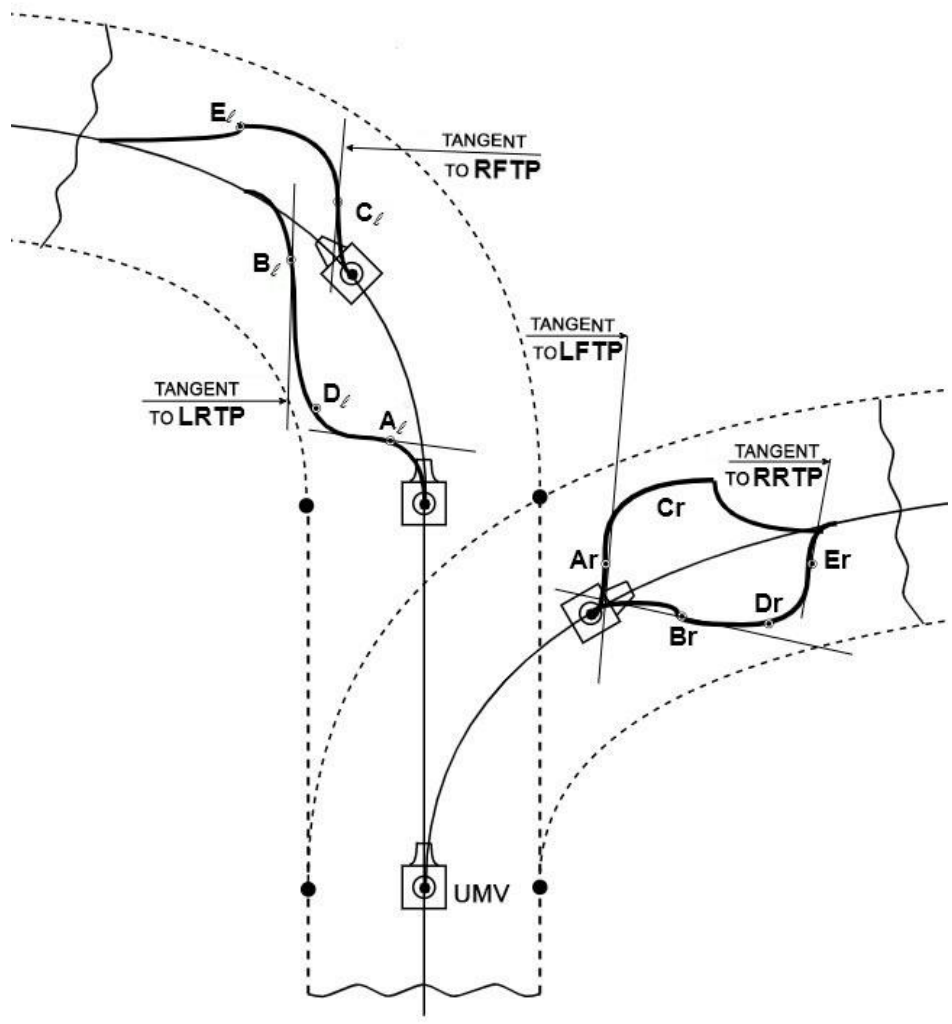

Fig. 2. Curved S-frames with CT leaving to the left and to the right of the nominal axial trajectory; combined view

On the fragment of the S-frame going along the curve to the right, $\mathrm{A}_{r}$ and $\mathrm{B}_{r}$ mark the inflection points of the left and right forward trajectories signals, and the point $\mathrm{E}_{r}$ marks the 
inflection point on the right reverse trajectory. The activation points of the modal control procedure are the moments $\mathrm{C}_{r}$ and $\mathrm{D}_{r}$ on the left and right trajectories, respectively.

Reverse TPs in modal control mode for this S-frame have the same character as for the S-frame with a left curvature.

For informatively complete and semantically transparent processing the ACSS- and ECSS-signals, fragments were introduced into the software complexes of the latter, providing the formation of the so-called wavelet functions $[15,16]$ and Cohen's class quadratic wavelet distributions [17-19].

Fig. 3 shows a view-reconstruction of a wavelet map (W-map), which in the format of Wigner time-frequency distributions $[16,19,20]$ shows the signal of the UMV CT leaving to the left of the NAT, and Fig. 4 - the signal for the UMV CT deviating to the right. In this case, the process of CT deviating to the left of the NAT is determined by decreasing the frequency of the generalized variable signal $x(t)$ specified in the CDS software and hardware complex, and CT deviating to the right of the NAT - by increasing.

In Fig. 3 , the moment $\tau_{l}$ corresponds to the beginning of the UMV CT deviation from the NAT under the influence of autonomous-sporadic disturbances. The character of the CTsignal is determined by the second-order aperiodicity and is formed of individual timefrequency atoms (TFAs) in accordance with the wavelet matching pursuit algorithm [16, $19,21]$. The parameters of the representation of this non-stationary process are used to determine the parameters of the upcoming mode - the mode of dynamic modal control. In addition, the form of the wavelet mapping of a signal $\mathrm{f}_{H O T}$ stationary in frequency is also shown here.

Fig. 4 demonstrates the time-frequency dynamics of the signal characterizing the UMV $\mathrm{CT}$ in stationary and non-stationary modes when the CT moves to the right of the NAT. Here, the moment $\tau_{r}$ is the moment when the CT starts moving away from the NAT.

The process of the UMV current trajectory deviation can be described with a twoexponential function of the transient response for the second-order aperiodic link:

$$
x(t)_{b / e i}=x(t)_{0 b / e i}+x(t)_{m b / e i} \sin \left[2 \pi f_{v a r}\left(t-\tau_{b}\right)-\varphi_{0}\right],
$$

where $f_{\text {var }}=f_{b}\left[1\left(t-\tau_{b}\right)\right]+\left(f_{e}-f_{b}\right)\left(\left[1\left(t-\tau_{e}\right)\right]-\exp \left[-\left(t-\tau_{b}\right) / T_{3}\right] T_{3} /\left(T_{3}-T_{4}\right)+\right.$ $\left.+\exp \left[-\left(t-\tau_{\mathrm{b}}\right) / T_{4}\right] T_{4} /\left(T_{3}-T_{4}\right)\right)$ - changing the frequency of the chirp signal [19] (frequency-time-dependent signal) determining the forward process of deviating the UMV trajectory from the NAT; 


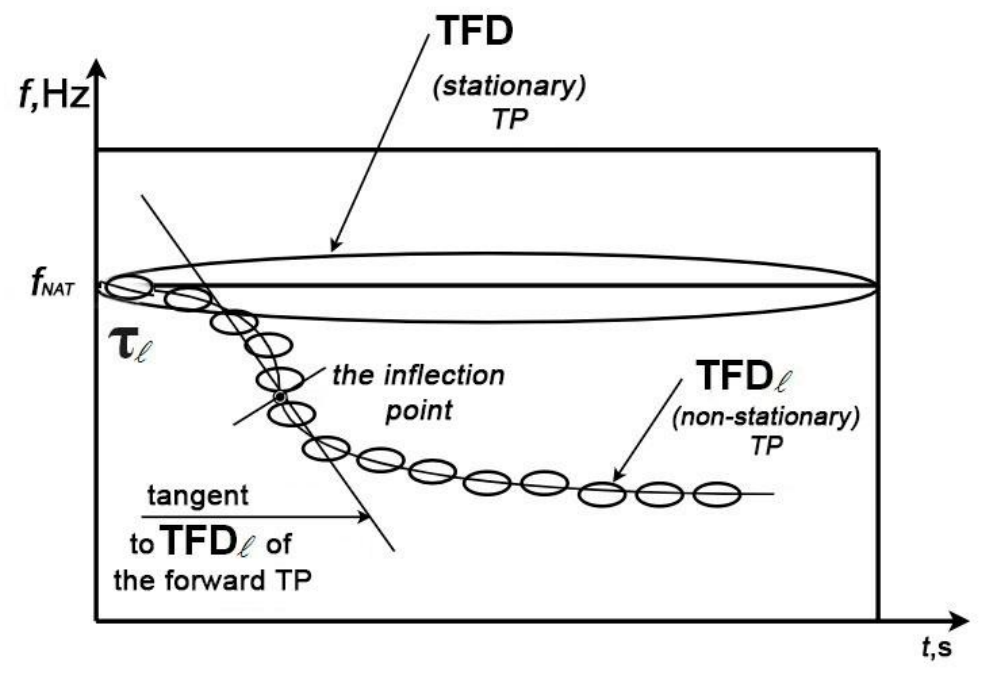

Fig. 3. W-map for stationary and forward non-stationary signals with decreasing instantaneous timedependent frequency; the latter characterizes the UMV CT leaving to the left of the NAT

$x(t)_{m b / e i}=x_{m b i}+t / T, \tau_{b} \leq t \geq \tau_{e}-$ changing the amplitude of the variable component of the signal;

$x(t)_{0 b} /_{\mathrm{ei}}=x_{0 b i}\left[1\left(t-\tau_{b}\right)\right]+\left(\mathrm{x}_{0 e i}-\mathrm{x}_{0 b i}\right)\left(\left[1\left(t-\tau_{e}\right)\right]-\exp \left[-\left(t-\tau_{b}\right) / T_{3}\right] T_{3} /\right.$ $\left(T_{3}-T_{4}\right)+$

$\left.+\exp \left[-\left(t-\tau_{b}\right) / T_{4}\right] T_{4} /\left(T_{3}-T_{4}\right)\right)$ - change in the aperiodic component of the forward process determining the deviation of the current trajectory from the NAT.

\section{TFDr}

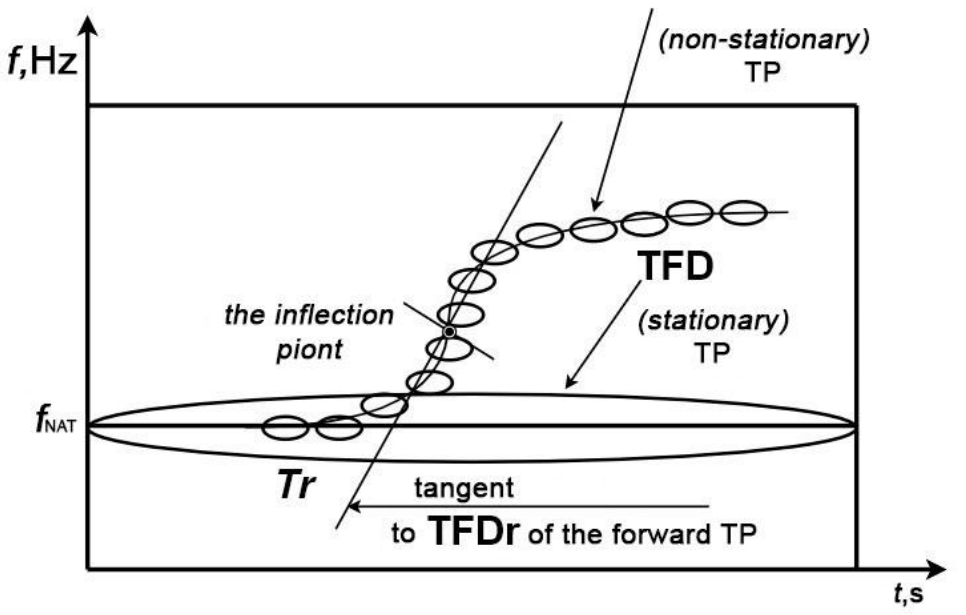

Fig. 4. W-map for stationary and forward non-stationary signals with increasing instantaneous timedependent frequency; the latter characterizes the UMV CT leaving to the right of the NAT 
Thus, the complete graph of transient processes for two cycles of the current trajectory deviation includes direct disturbed and reverse restoring processes.

\section{Results}

The article presents the solution of a complex problem of developing planned routes for the movement of unmanned vehicles in a quarry, models of routes within the framework of the so-called S-frames, which is relevant in scientific and applied significance.

The structural position of the software package as part of the computer-aided dispatching system within the global "Smart Quarry" management structure (SQMS) is described.

The functional significance of the computer-aided dispatching system (CDS) developed as a fragment of the control center (CC), which is part of the SQMS system, is considered.

Information on the nature of the UMV planned routes is given.

In addition, the data are given that allow designing a computer-aided system for modal control of the UMV non-stationary movement along the routes developed using cartography systems.

Information is given on the mathematical formalization of time signals characterizing forward and reverse transient processes, which determine the dynamics of UMV current trajectories on working routes.

Schematic graphs of transient processes in one-dimensional format and in multidimensional form (as wavelet distributions) are presented; this allows for ongoing processing the signals indicating the UMV movement in a semantically transparent and information-rich form.

The solution of the described complex problem is of significant scientific and practical interest for the country's economy, in particular, for the mining industry, and can be claimed and effectively used both in the mining industry and in road design for urban and rural areas when road markings are not available, and also in other industries.

\section{Conclusions}

The main theoretical and practically significant results of the work and conclusions are presented below.

1. Two approaches are described for obtaining images of locations, which make it possible to create frame models of both local routes for UMVs and the state of mining equipment in general.

2. A draft description of the characteristics of control functions incorporated in the autonomous and external control subsystems (ACSS and ECSS) is proposed.

3. The use of a wavelet transforms technique has been introduced to the concept of forming the models describing the UMV movement along current routes - for recording and displaying (at the video graphic level) a complex of signals characterizing the UMV dynamics in the conditions of stripping / mining operations.

The work is performed with the financial support of the Ministry of Science and Higher Education of the Russian Federation under the agreement No 075-11-2019-034 dated 22.11.2019 with PJSC KAMAZ on the integrated project "Development and creation of high-tech production of autonomous heavy platforms for unmanned mining of minerals in the "Smart pit" system, with participation of T.F. Gorbachev Kuzbass State Technical University in terms of research, development and technological works. 


\section{References}

1. D.M. Dubinkin, Mining Equipment and Electromechanics. 4, 59 (2020)

2. D.M.Dubinkin, V.Yu. Sadovets, I.S.Syrkin, I.V. Chicherin Mining Equipment and Electromechanics. 6, 25 (2020)

3. I.V. Chicherin, B.A. Fedosenkov, I.S.Syrkin, V.Iu. Sadovets, D.M. Dubinkin Gornyi zhurnal. News of the Higher Institutions. Mining Journal. 8, 109 (2020)

4. B. L. Golden, D. R.Shier, Networks. An International Journal, 72, 4, 409 (2018).

5. H. B.Richard, W.Shyy, Encyclopedia of Aerospace Engineering (John Wiley \& Sons, Ltd., New York, 2010)

6. K.Valavanis, G. J. Vachtsevanos, Handbook of Unmanned Aerial Vehicles (Springer Reference, 2015)

7. Tsach, Shlomo, Tatievsky, Alex, London, Lior, Unmanned Aerial Vehicles (John Wiley \& Sons, Ltd., New York, 2010)

8. Swierczynski, Slawomir, Felski, Andrzej. 2019 ENC. IEEE

9. Agisoft Photo Scan Professional Edition. - User Manual - v. 1.2: http://www.agisoft.com/pdf/photoscan-pro_1_2_ru.pdf

10. E.A.A. Abdulla, Z.M. Fadlullah, H. Nishiyama, N. Kato, F. Ono, and R. Miura, Proc. IEEE INFOCOM, 736 (2014)

11. Zengin, Ugur, A.Dogan, IEEE Transactions on Robotics 23.2, 294 (2007)

12. Goodwin, Graham C., Control System Design (Pearson Education, Inc., Prentice Hall, New York, 2001)

13. V. Streitz, Method of the state space in the theory of discrete linear control systems (Nauka, Main edition of physical and mathematical literature, Moscow, 1985)

14. B.A. Fedosenkov, Automatic control theory. Classic and modern sections: Train. Manual (Kemerovo State University, Kemerovo, 2018)

15. S. Mallat, Wavelets in signal processing. (Mir, Moscow, 2005)

16. B.A. Fedosenkov, D.B. Fedosenkov, Computer-aided control of technological processes in a wavelet medium: Monograph (T.F. Gorbachev Kuzbass State Technical University, Kemerovo, 2021)

17. L . Cohen, Time-frequency analysis (Englewood Cliffs, Prentice Hall, New York, 1995)

18. D.B. Fedosenkov, Steel in Translation, 49, 4, 252 (2019)

19. S. Mallat, A wavelet tour of signal processing (Academic Press - 2nd Ed., New York, Ecole Politechnique, Paris; Courant Institute, New York, 1999; Reprinted, 637 pp. (2001); 3d Edition. The Sparse Way. Academic Press, 832 pp. (2008)

20. L. Cohen, IEEE Trans. Acoustic, Speech, Signal Processing, ASSP-35, 6, 796 (1987)

21. SE Ferrando, Signal Processing, 80, 2099 (2000) 